Wearable technology for posture monitoring at the workplace

Peer-reviewed author version

Bootsman, Rik; Markopoulos, Panos; Qi, Qi; Wang, Qi \& TIMMERMANS, Annick

(2019) Wearable technology for posture monitoring at the workplace. In: International Journal of Human Computer Studies, 132, p. 99-111.

DOI: $10.1016 /$ j.ijhcs.2019.08.003

Handle: http://hdl.handle.net/1942/29520 


\title{
Wearable technology for posture monitoring at the workplace
}

\author{
Rik Bootsman, Panos Markopoulos, Qi Qi, Wang Qi, \\ Department of Industrial Design, Eindhoven University of Technology
}

Annick AA Timmermans

Faculty of Rehabilitation Sciences, University of Hasselt

\begin{abstract}
Prolonged strenuous postures in occupational context may lead to low back pain. Avoiding such occurrences is known to help prevent low back pain episodes or may contribute to recovery. This research concerns wearable sensing technology to support posture monitoring for the prevention of occupational low back pain and, more specifically, how smart garments can help nurses avoid prolonged strenuous postures at work. We introduce BackUp, a system comprising of a smart shirt connected to a smartphone application that provides feedback and advice on low back posture, and we describe its design and implementation. We report on a series of studies that contributed to its development: an anthropometric study $(\mathrm{N}=60)$ to decide on the placement of sensors on the lower spine; a brief field study aimed at evaluating user experience and attitudes towards the shirt $(\mathrm{N}=17)$, and a second field study intended to assess its effectiveness in helping nurses avoid prolonged strenuous postures at work $(\mathrm{N}=13)$. These studies demonstrate how smart clothing can support posture feedback in real life conditions. While the results from the field studies are encouraging regarding the potential of this technology, further research is needed to establish the durability of the behaviour modification achieved through smart garments.
\end{abstract}

\section{KEYWORDS}

Smart garments; interactive clothing; posture correction; low back pain; persuasive technology, nurse, user test, awareness. 


\section{INTRODUCTION}

Roughly a decade has elapsed since the introduction of commodity level activity trackers such as the Fitbit $^{\mathrm{TM}}$. Currently there is a thriving industry and a fast-growing research field concerned with the development of wearable systems for personal health monitoring. The majority of available devices are wrist worn, placed inside pockets, or clipped onto clothing. As electronics are further miniaturized and as e-textiles are becoming increasingly available, it also becomes more feasible to integrate electronics into 'smart' garments to support healthy living and rehabilitation. This emerging application area presents opportunities and corresponding challenges pertaining to industrial design, interaction design and ensuring the potential effectiveness of these solutions. This paper presents a design driven investigation that combines these perspectives for the design and evaluation of BackUp; a smart garment to support occupational low back pain prevention for nurses.

Low back pain is widespread; the lifetime prevalence of low back pain in the developed world ranges between $60-70 \%$ and is a major cause of activity limitation and work absence worldwide [24]. Costs relating to reduced productivity of workers and to treating low back pain are high; for example, Wening et al [37] estimate an average cost of $€ 1322$ (95\%) per patient per year in Germany. Such observations motivate the development of technologies to help prevent and treat low back pain.

$90 \%$ of the low back pain cases are non-specific, demonstrating no identifiable pathology. However, low back pain is often associated with one's profession. A recent survey among hospital nurses reported $48.8 \%$ prevalence in the week prior to the survey and $60.9 \%$ in the six months prior to the survey [3]. The risk on low back pain increases due to prolonged awkward postures or movements [17] and is often associated with repetitive adverse postures in professional workers such as nurses [12] and assembly workers [25]. Specifically, repetitive flexion is known to be a potential cause of impaired spinal muscle control [23] that may result in low back pain. Strategies to limit these contributing factors can potentially help to alleviate symptoms, disability and prevent recurrence [31]. Therefore a key element of both prevention and treatment of low back pain is to vary posture and to avoid prolonged strenuous postures that may contribute to pathogenesis and sustainment of pain [24]. 
Posture correction pertains to different positions such as sitting, standing, and lying down, as well as postures related to one's work, e.g., bending, lifting, etc. There is no unequivocal definition of what a correct and ideal posture is.

Instructing workers with low back pain on posture is not enough to help them to change postural behaviour, as it is known that intrinsic feedback mechanisms are impaired in persons with low back pain [5] making it difficult for them to be aware of and to change or avoid adverse postures. For this reason, extrinsic feedback is required which can be provided by the therapist or by suitable technological aids. O'Sullivan [22] found that postural feedback can reduce low back pain within a single session, though long-term treatment and effectiveness of such feedback by the therapist have not yet been investigated. Furthermore, even if people understand which posture may give rise to their discomfort or pain, they need to remember to change it and here posture tracking technologies may play a useful role.

Wearable and pervasive technology can be applied for tracking posture unobtrusively during their daily life; this can be of benefit for persons whose job involves repetitive or prolonged strenuous postures . Despite these positive prospects there are still too few self-tracking technologies for posture monitoring that address the prevention and treatment of low back pain. So far, there are only a few research efforts to develop posturemonitoring solutions for the low back and there is yet limited evidence reported as to their effectiveness; we review such work in the following section.

This research set out to develop a smart garment that can help nurses avoid prolonged and strenuous postures at work. We developed BackUp a posture monitoring system that combines a smart sensing garment with a smartphone application. The garment design focused primarily on ensuring comfort, unobtrusiveness, sufficiently accurate placement of the sensors and reliable detection of poor lumbar posture; the application concept design focused on identifying an appropriate feedback strategy for changing the posture related behaviour of the user.

In the following sections we review related work and then introduce the BackUp system concept. We then go on to describe two field studies that examine nurses' attitudes towards wearing BackUp and using it as a posture monitoring system and we provide initial evidence as to its potential in ensuring correct posture at work. We end with a discussion of general challenges pertaining to the design of smart garments for posture monitoring. 


\section{TECHNOLOGIES FOR POSTURE MONITORING AND CORRECTION}

A range of technologies have been developed to monitor posture and provide feedback, however the majority of these fall outside the scope of this article as they concern patients with balance problems or parts of the anatomy that do not relate to low back pain. On the other hand, technologies developed specifically for low back pain prevention or treatment, are few and far between. A recent systematic literature survey of technologies for upper body posture and movement monitoring [36] located only two studies concerning the low back: Specifically, Bhomer et al [2] present a concept prototype which uses textile sensors to provide sonified feedback regarding low back posture, and Newbold et al [21] demonstrate how music based sonification can provide range of movement feedback during rehabilitation training. Both these works focus on interaction design issues and do not examine either the accuracy or the effectiveness of their solution. Moreover, Newbold et al [21] do not focus on low back pain prevention as such; rather they consider low back posture cursorily only as an example to help illustrate their sonification concept. More specifically, accelerometer data obtained from a smartphone mounted on the low back is transformed to represent the extent and duration of stretching exercises in musical patterns. In both these concept designs, posture tracking is not intended for use during daily life activities, but is intended as an aid during rehabilitation therapy sessions.

Quite early on, Veltink [33] illustrated the feasibility of posture measurement with accelerometers; a low pass filter was used to eliminate measurements during movements taking into account only steady data signals to identify relatively static user postures. To discriminate between different postures, several sensors were attached at different parts the body. Concerned with developing a measurement approach Veltink [33] did not integrate these sensors into an integrated interactive system that would provide feedback to users. Wang et al [35] use accelerometers to support thoracic posture correction during rehabilitation; they emphasize how attention must be paid to place sensors accurately and fit them close to the body to prevent measurement artefacts resulting from deformations or movements of the garment.

A commercially available posture monitoring device for low back pain prevention is the Lumo Back ${ }^{\mathrm{TM}}$, which is worn as a belt under clothes and which signals deviations from an upright posture with a vibration. While this device is an elegant wearable appliance, it does not measure lumbar posture as such. Its principle of operation can 
potentially result in false alarms notifying users every time they lean forward even when their lumbar posture is correct, which may undermine the credibility of the device and reduce compliance to its feedback. So far, there has been no evidence published regarding how users experience Lumo Back and whether they can improve their posture by wearing it.

The SpineAngle [27], an experimental device aimed at preventing low back pain, was user tested. This device measures the pelvic angle with accelerometers placed on a belt at the level of the pelvis as a way to estimate lumbar spine posture. Ribeiro et al evaluated the SpineAngle in a quasi-experimental study with 29 participants over 6 weeks, demonstrating a significant reduction in flexed posture compared to the initial baseline measures, when participants were provided with constant feedback (a notification every time a flexed low back posture is detected). However, this study did not find any differences to a control group who received no posture feedback at all [27]. Interestingly, Ribeiro et al, [27] report that users thought the device helped correct their posture, even though this was not really the case. Nevertheless, the idea of using wearable sensors to measure the lumbar angle rather than the overall inclination of the body is promising and is adopted also in our own design concept.

Feedback design is of key importance in avoiding prolonged strenuous postures to reduce symptoms of lower back pain. Dekker et al [9] showed that personalized feedback messages have additional value over continuous visual feedback in stimulating patients with chronic low back pain to vary their activity in order to avoid straining their back. Zheng and Morrell [39] explored how vibrotactile haptic feedback from sensors integrated in a chair could provide guidance on sitting posture, and demonstrated its effectiveness in guiding posture. A comparison with other feedback modalities showed that a peripheral embodied display was the most acceptable form of feedback. Van Almkerk et al [1] developed a sensory chair that helps users maintain posture awareness unobtrusively through visual feedback (a table application with real time and summary feedback) and haptic feedback (a pillow inflates to encourage lumbar extension) intended as a peripheral display [13]. A preliminary evaluation with four participants indicated positive expectations towards the system. There have been similar attempts to integrate posture sensing into chairs; however these solutions only provide feedback on sedentary posture (as opposed to while a person moves, bends, lifts) and are only useful as long as the user is sitting on the sensory chair, which inevitably will be but a small portion of the day. This limits the potential applications and 
use of this device to office environments. To this point there have been no attempts to develop posture monitoring appliances for occupational low back prevention for other forms of work.

The Valedo ${ }^{\text {TM }}$ system by Hocoma (www.valedotherapy.com) uses inertial sensors that a therapist can adhere against the skin on the top and bottom of the lumbar spine area of the patient; the sensors are used as an input device for video games that encourage exercising pelvic tilts and motor control of the lumbar spine during movement of the extremities. The Valedo ${ }^{\mathrm{TM}}$ is not targeted at posture tracking and correction throughout the day; the latter which would require addressing practical issues such as, skin irritation, letting patients control when they are tracked or not, and letting them place devices independently in case they are detached.

There are other posture monitoring devices that are not intended for ambulatory and independent use in a real-life context. A good example is the diagnostic medical tool for measuring spine curvature by Epionics [7]. It is adhered to the back and can be worn for up to 24 hours, and thus does not lend itself for self-tracking and behaviour modification purposes.

An ambulatory solution is the BodyGuard ${ }^{\mathrm{TM}}$ device which has been used in research studies to measure low back curvature of cyclists [32]; an expert is expected to place this device and adhere it on the skin, so this also is not practical for everyday independent use. On the positive side, O'Sullivan et al. report an experiment where BodyGuard was found effective in providing corrective posture feedback on seating posture [22].

ViMove $^{\mathrm{TM}}$ [15] (by dorsavi.com) combines a motion-sensor mounted on the thoraco-lumbar junction, another one mounted on the upper sacrum to track lumbar spine and pelvic components movements, and an EMG patch to capture muscle activity. ViMove allows personalized tracking regimes to be defined by the therapist to identify situations such as over- and under-activity of lumbar musculature, as well as prolonged sitting in poor posture. Kent et al [15] report a randomized clinical trial with low back patients that demonstrated the effectiveness of a 10 week long treatment, which combined 6 consultations with a clinician with self-tracking using ViMove for 3-4 hours a day. Kent et al focused on the effectiveness of the combined treatment regime but did not study the effectiveness of the device in helping people self-track and avoid sustained strenuous postures. Both BodyGuard and ViMove have the disadvantage that they have to be attached to the patient's skin with tape, 
which may lead to skin irritation in persons who are sensitive, limiting their suitability for long-term use in daily life.

All applications reviewed so far provide range of motion feedback directly to their user. A different approach is taken by Shin et al [29] who examined posture correction for office workers as a case of behaviour modification where they apply and evaluate their relational norm intervention model. According to this model a negative feedback is delivered to 'helpers' (initially they receive a notification and eventually their phone is locked) when the monitored user fails to demonstrate the target behaviour (in this case they have a poor sitting posture). Shin et al demonstrated empirically that this behaviour change approach is more effective than simple notifications. However, the strong element of coercion in the relational norm intervention model and the compliance of one more person that is required (that the helper accepts to receive the negative feedback for the behaviour of the targeted user) limit its applicability outside the context of behavioural experiments. For the purposes of their

study, Shin et al used a single point measurement to detect deviations from an upright position (in the same way as in the Lumo Back discussed above).

In conclusion, there is as yet no garment-based posture monitoring and correction device that can be used for sustained periods, that can provide accurate feedback of lumbar back posture and that has been shown to be effective in helping people maintain a correct posture.

\section{DESIGN OF BACKUP}

BackUp is a T-shirt worn under the nurse uniform adorned with sensors to track lower back posture. A connected smartphone application provides notifications about sustained lumbar flexion postures and can provide tips for changing posture. We designed BackUp in an iterative process in which we involved nurses as informants in the early exploration phases and later on as test-participants. Initially we interviewed nurses regarding their work and low back pain prevention and regarding their attitudes towards wearable solutions. We also let them try out the Lumo ${ }^{\mathrm{TM}}$ device to gain insight into how they would experience a wearable low back posture tracking device. We then created an early prototype of BackUp that could demonstrate the sensing functionality. Based on feedback provided by nurses, physiotherapists, ergonomists and clothes manufacturers we implemented a more refined prototype that was sufficiently robust and comfortable to allow evaluation in two field studies which 
focused respectively on user experience aspects and on the effectiveness of the solution for posture correction. We detail this process below.

\subsection{Initial design development}

To understand how users experience posture tracking we asked 6 nurses in a neighbouring hospital to wear Lumo Back for 4 days during work hours and to consult the device a few times during the first three days; on the last day we encouraged them to use it in their own way. During this period nurses kept a brief paper diary where they would record impressions and improvement suggestions about Lumo Lift at the end of each day. At the end of this period we interviewed them regarding their experience with the device.

In these debriefing interviews, nurses were generally positive towards wearing a posture monitoring system but mentioned that they had started to ignore its notifications already from the first day, and all of them would ignore it on the last day. The reasons they did so were mixed; sometimes notifications would arrive at an inconvenient moment, sometimes nurses did not take the feedback seriously assuming it was a false alarm or even after noticing a notification they would simply ignore it or forget it. The explanations they offered for not taking the notifications seriously were that they were absorbed in their tasks, they received too many notifications or that Lumo produced false alarms when bending down, even while keeping a good posture, and false negatives when one's torso is upright, but has a poor lumbar posture. Another comment was that they did not always know how to act on the notification they received in order to improve their posture. We concluded that it is necessary to provide a more accurate detection of sustained strenuous posture events and to elaborate the behaviour change feedback with some advice as to how to improve their posture.

To measure the lumbar posture accurately through the day at different locations in the hospital and while nurses engage in different tasks a wearable solution offers substantial practical advantages compared to approaches such as sensor-equipped chairs, off body camera based systems, etc. These systems can be effective but only when the user is in the selected location and position (sitting on the chair) or standing/sitting within the view of the camera. On the other hand, the wearable solution should be comfortable and should not hinder nurses in their work. As nurses move through the day, they do not maintain a constant upright posture. However, leaning/bending forward or backward is not an indication of poor posture; rather it is a sustained flexion of the 
lower back that may be a problem. For this reason, we decided to place sensors at the two vertebrae (L1 and L5) that define the lumbar spine area at its two ends in order to estimate its curvature (see figure 1).

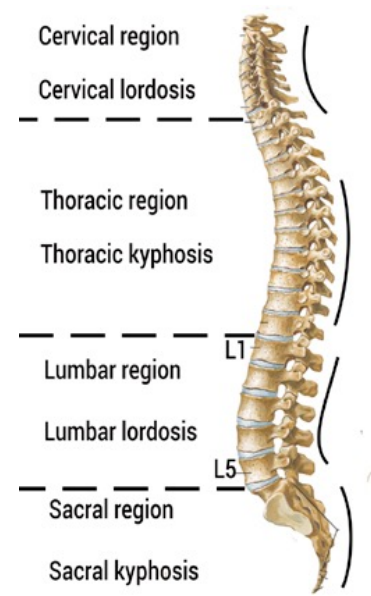

Figure 1. The lumbar region of the spine includes five vertebrae, which are labelled L1 to L5. The angle between L1 and L5 provides an indication of the curvature of the lumbar spine. (Source of spine image: http://www.imaios.com)

Sensor placement on a garment needs to be reconciled with requirements for comfort, aesthetic appearance, and ease of use. Initially, we attempted to place sensors on a band that would be worn around the waste. However, some first explorations showed that while the band could create a tight fit around the body, after wearing it for a little time the sensors would slide both vertically and horizontally away from their intended location. We then decided to put them on a T-shirt instead and developed a prototype (see figure 2), which we could demonstrate to several stakeholders and experts. During this stage we first attempted to connect the printed circuit board to the soft fabric using tin plated brass snaps. However, connections were not reliable enough and the distance from the sensor to the skin increased as a result of their physical size. The pouch solution also proved impractical for changing batteries and for trouble shooting. To ensure a good fit of the sensors we attached elastic bands around the low back, which however could make it difficult for nurses to put the shirt on and take it off. 


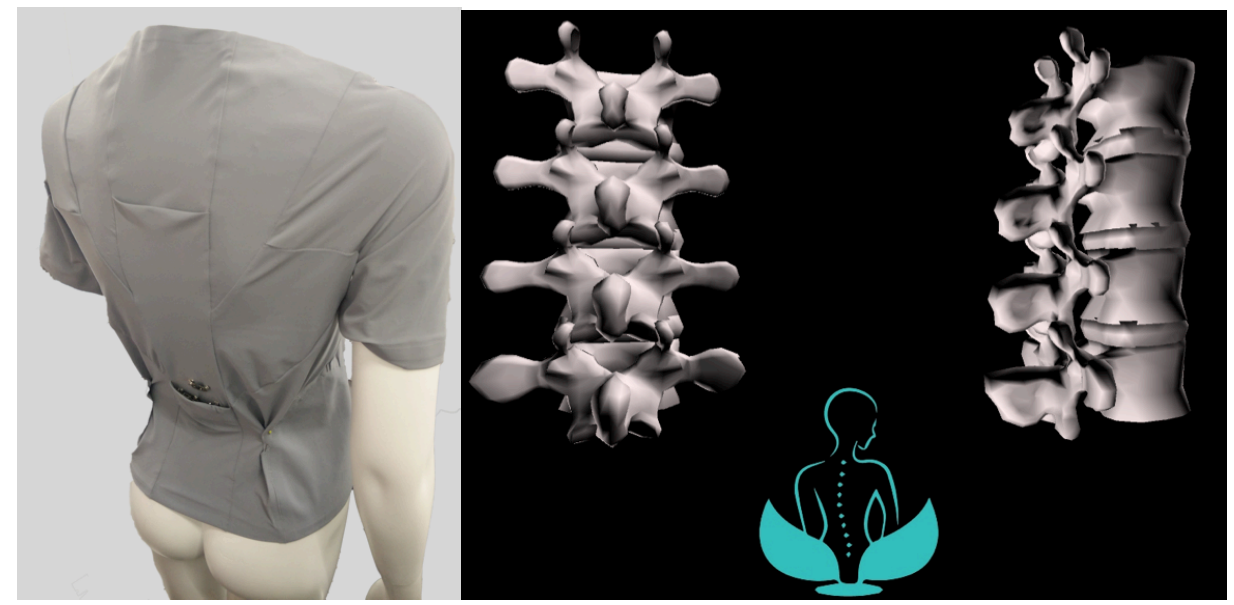

Figure 2. Initial feasibility prototype of BackUp. Left: the first version of the t-shirt with electronics hidden in a pouch at the back. Right: Animation of the lumbar used to demonstrate the concept to stakeholders.

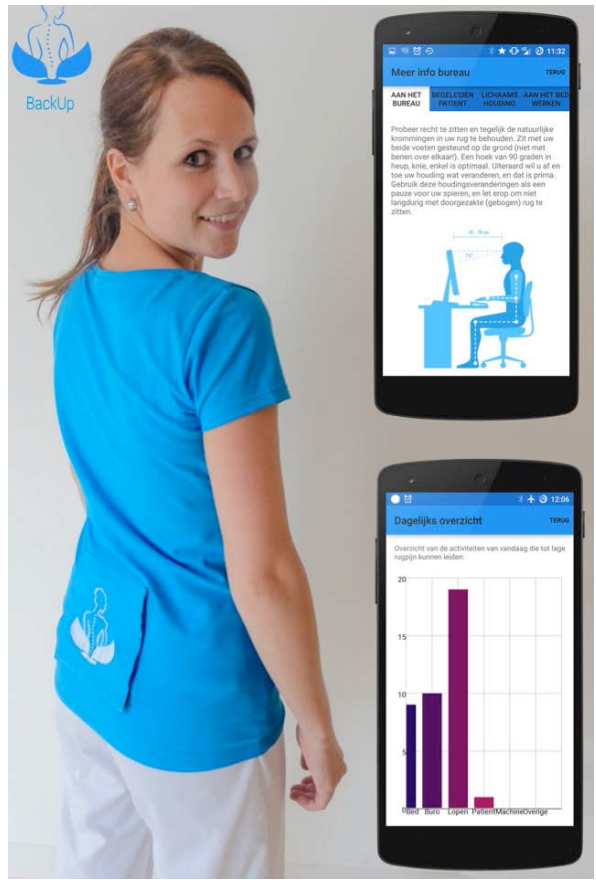

Figure 3. BackUp a smart shirt for tracking low back posture and the feedback it gives via a smartphone.

\subsection{Improved BackUp Shirt}

The major objective in designing the BackUp shirt has been to provide sufficient comfort to wear through the day and postural feedback for users. BackUp is completely standalone, and battery operated. All electronics are washable, except of the battery. 
To measure the orientation of the lumbar two Inertial Measurement Units (IMU) with 9 degrees of freedom were used to register the movements of the spine on vertebrae L1 and L5. The IMU's are the LSM9DS0 sensors by STMicroelectronics, which we chose because of their accuracy and small footprint, and because there is a printed circuit board that can be easily sewn onto textiles. The IMU's are connected to a wearable microcontroller, the Adafruit Flora, via I2C. We chose the Flora, because one can use the Arduino programming platform (www.arduino.cc) and it is easy to sew on clothing. It is thinner than the more widely used Arduino Lilypad, which also does not have a battery connector.

We incorporated the sensors in a tight-fitting shirt to be worn under the normal nurse uniform (figure 3 ) to keep them at their intended position. We used a Bluetooth Low energy module (HM10) to communicate wirelessly with an Android smartphone. HM-10 is a small and low-cost Bluetooth breakout chip, which is easy to address via UART (serial communication). A $500 \mathrm{~mA}$-h lithium polymer battery was used to power the circuit. The sensors and microcontroller are connected via an embroidered circuit of conductive thread in the shirt to avoid having hard wires running over the length of the back. Together with a clothes manufacturer we selected fabrics that could withstand the high washing temperatures used in hospitals and materials that would pass hygiene requirements. The electronics were embedded in the shirt via an embroidery process with conductive thread using machines suitable for large-scale production. Finally, we optimized the electronic circuit pattern for embroidering, e.g. introducing wider clearances between circuit paths and short lines between components.

We used an insulated conductive thread to allow connections to cross over each other resulting in a multi layered circuit design. The embroidered pattern we created in this way (see figure 4a) connects all hard circuit boards via conductive thread. In the improved BackUp prototype, an analogue switch (NX3L1T3157) connected the SDA pin of the microcontroller and the SDA pins of the two sensors; by scheduling the communication with the sensors we could access both sensors using the same pin of the microcontroller. An elastic material ensured a good fit at the lower back; this makes it harder to put on and take off the shirt than a casual cotton T-shirt, but it also makes BackUp stretch and fit snuggly around the body.

Besides the requirements for placement and accuracy of the sensing technology also the ability to wash the garment is important. With the exception of the battery that needs to be removed for washing, all components 
used, e.g., the microcontroller, the sensors and conductive thread are machine washable. Clothing for a hospital environment needs to survive 130 wash cycles and the goal is that BackUp should be able to achieve this as well. However, further testing and iterative improvements of the garment are needed before this can be achieved reliably.

High Conductivity Silver Plated Nylon thread 117/17 2ply was embroidered onto the shirt to create a conductive pattern on which the components could be connected (figure 4a). The conductive thread has to be handled carefully to ensure a low resistance circuit. Figure 4(b) shows all components placed on the conductive pattern; cotton cloth between the pattern and the components prevents shortcuts in the circuit while wearing the shirt.

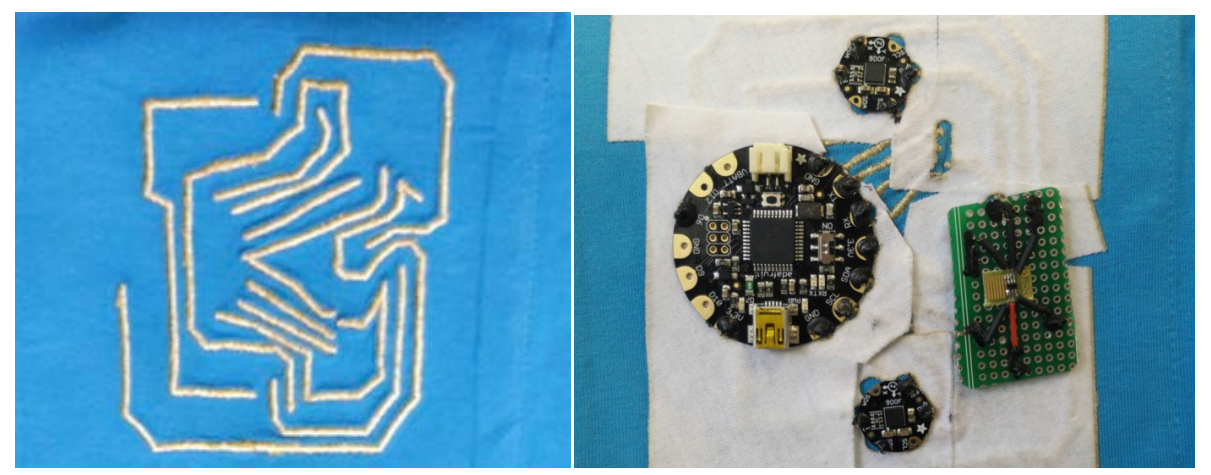

Figure 4 (a) Embroidered circuitry (b) placement of sensors, sensors (smallest circles), microcontroller, large circle and the switch breakout (green board)

\subsection{Sensor Placement}

In order to place sensors on clothes that can be mass manufactured (rather than tailor made to the body of the patient) and that users can wear without the help of a professional to position them, we first set out to establish whether a few different sizes of garments can ensure accurate enough positioning of the sensors for people of different size. In order to identify the principle by which we would approach the differential positioning of sensors for people with different sizes, we carried out a cross sectional study (reported in [30]) for adults (18-60 years old) where we measured intervertebral distances for persons with different anthropometric characteristics. 
Two independent assessors (senior physiotherapy students) marked bony landmarks of the spine C7, T4, T12, L1, L5, and Spinae Iliacae Posterior Superior (SIPS) (see figure 5); a third assessor evaluated discrepancies. Assessors measured intervertebral distances using a flexible ruler and registered full body length. Participants tried T-shirts of six different sizes (ranging from extra-small to extra-extra-large) and selected the size fitting them the best.

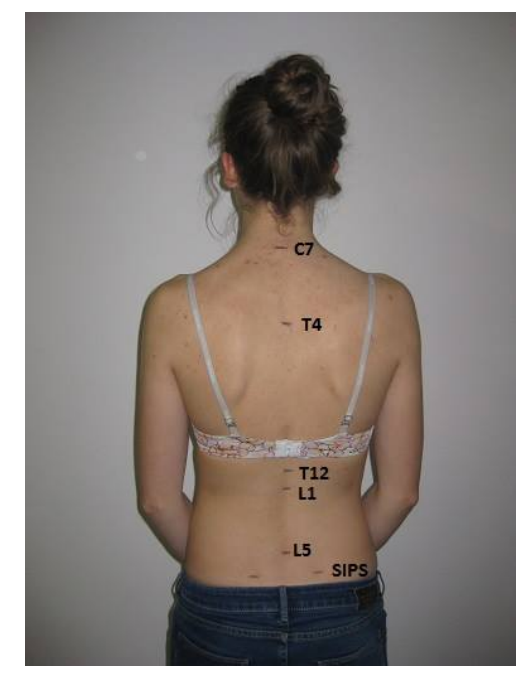

Figure 5. Bony landmarks on the spine

We analysed data using SAS JMP Pro, treating trunk length (distance between C7-SIPS) and full body length as independent variables after converting these measures to nominal data corresponding to equal intervals for each category (see Table 1). Because the data for the distance L1 to L5 was not distributed normally we applied a Kruskal Wallis test. We then made multiple comparisons using Wilcoxon signed ranks test for the distance L1L5.

Sixty people took part (18 male/42 female; with an average age of40.57 ( $\mathrm{SD}=14.55)$. We found significant differences for the intervertebral distance L1-L5 for persons with different trunk length $(\mathrm{p}=0.0041)$ and for persons with different full body length $(\mathrm{p}=0.0152)$ but not for persons with different $\mathrm{T}$-shirt sizes $(\mathrm{p}=0.12)$. Subsequent multiple comparison analyses showed significant differences in intervertebral distances in different categories for trunk length, full body length, and to a limited extent for T-shirt size.

From this analysis we concluded that we could determine the placement of sensors on L1 and L5 based on defaults chosen based on the trunk length rather than on the size of clothes somebody normally wears (e.g., small, 
medium, large). However, the distance from C7-L1 cannot be accurately predicted based on the trunk length or full body length. Thus, we opted for making standardized patches where the distance between two sensors is fixed for different ranges of trunk length but where the exact height at which the patch will be placed on the T-shirt can be adjusted. In our studies described in later sections of this paper, a physiotherapist could point out for any specific patient, at exactly which of a few set positions on the T-shirt the patch should be placed.

\begin{tabular}{|c|c|c|}
\hline & & L1-L5 M(SD) \\
\hline \multirow{6}{*}{ T-size } & $\mathrm{XS}$ & $7.57(1.05)$ \\
\hline & $\mathrm{S}$ & $7.77(1.42)$ \\
\hline & $\mathrm{M}$ & $8.27(1.54)$ \\
\hline & $\mathrm{L}$ & $8.39(0.66)$ \\
\hline & $\mathrm{XL}$ & $8.30(0.70)$ \\
\hline & $\mathrm{XXL}$ & $9.50(1.73)$ \\
\hline \multicolumn{2}{|c|}{ P-value (F-test or Wilcoxon/Kruskal-Wallis) } & 0.12 \\
\hline \multirow{4}{*}{ Trunk Length } & $\mathrm{A}(42.3-46.2 \mathrm{~cm})$ & $7.26(1.41)$ \\
\hline & $\mathrm{B}(46.3-50.2 \mathrm{~cm})$ & $7.75(1.06)$ \\
\hline & $\mathrm{C}(50.3-54.2 \mathrm{~cm})$ & $8.57(1.24)$ \\
\hline & $\mathrm{D}(54.3-58.2 \mathrm{~cm})$ & $9.40(1.29)$ \\
\hline \multicolumn{2}{|c|}{ P-value (F-test or Wilcoxon/Kruskal-Wallis) } & $0.0041 * *$ \\
\hline \multirow{4}{*}{ Full Body length } & A $(152.0-161.9 \mathrm{~cm})$ & $7.68(1.27)$ \\
\hline & $\mathrm{B}(162.0-171.9 \mathrm{~cm})$ & $7.64(1.31)$ \\
\hline & $\mathrm{C}(172.0-181.9 \mathrm{~cm})$ & $8.01(1.13)$ \\
\hline & $\mathrm{D}(182.0-191.9 \mathrm{~cm})$ & $9.60(1.26)$ \\
\hline \multicolumn{2}{|c|}{ P-value (F-test or Wilcoxon/Kruskal-Wallis) } & $0.0152 *$ \\
\hline
\end{tabular}

Table 1. Average intervertebral distances for persons with different T-shirt size, trunk-, and body length $\left({ }^{*} \mathbf{p}<0.05,{ }^{* *} \mathbf{p}<0.01\right.$, $* * * \mathbf{p}<0.001)$, adapted from $[30]$ 


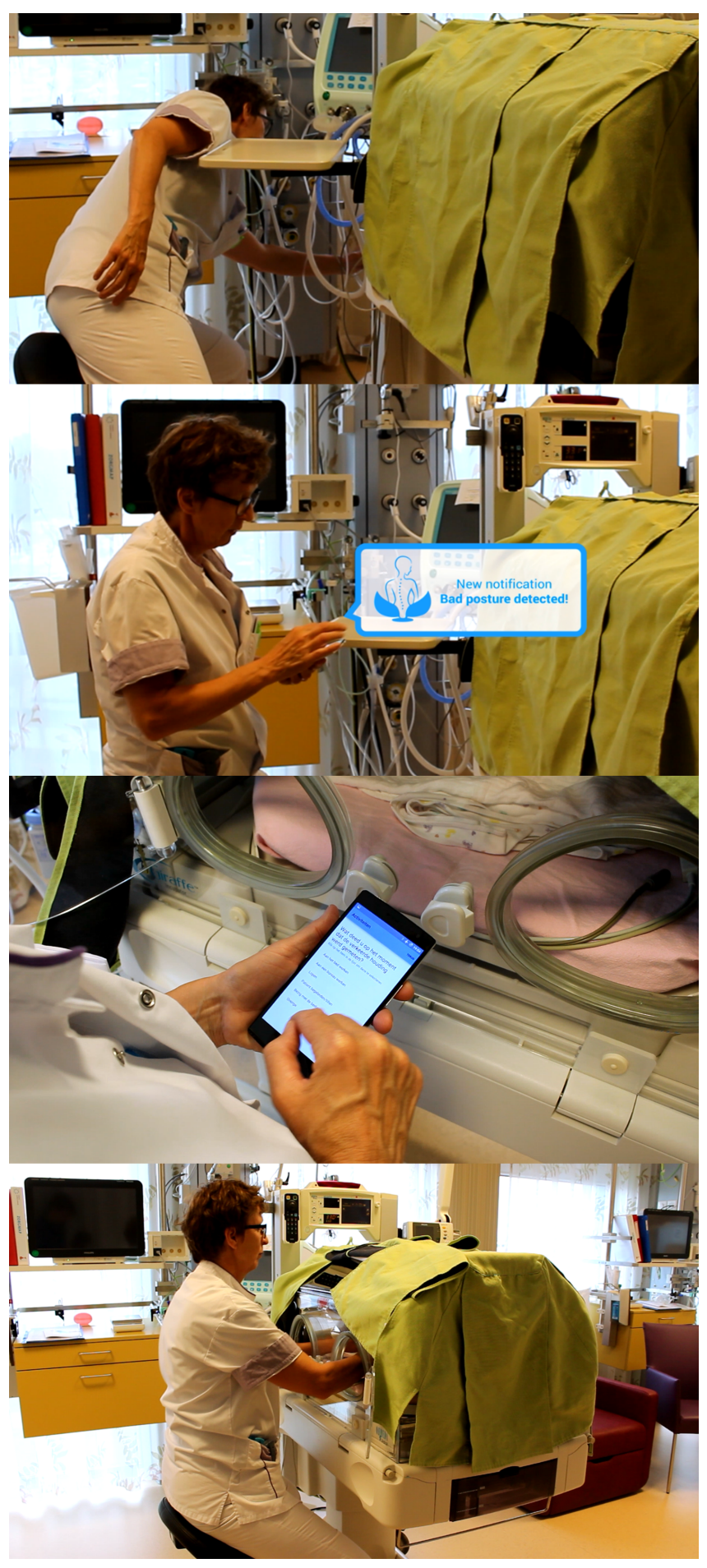

Figure 6. Interaction with the system in sequence: The shirt detects a sustained flexion of the lower back and generates a notification, the nurse can open the notification and fill in the activity she's working on and closes the app before continuing with her work. 


\subsection{Software}

We implemented the posture tracking and feedback application on Android (www.android.com) and Arduino. An AHRS sensor fusion algorithm handles data it receives from the shirt locally, converting readings from the accelerometer, gyroscope and magnetometer to calculate 3D orientation. It sends converted data continuously to the connected Smartphone, which compares to threshold values representing acceptable postures.

Because people have different body shapes there is no single set of values settings that reflect an ideal curvature of the lumbar spine. Therefore, the application can be personalized independently or with the help of an expert, e.g., an ergonomist or physiotherapist, who can identify what is a 'neutral' position for the user. The calibration procedure simply averages sensor readings over a 5-second interval when the user maintains an upright posture; nurses can easily maintain this posture for 5 seconds, so we did not attempt optimize the duration of the calibration procedure.

The application runs in the background waiting to receive data via Bluetooth. When the application receives data, it checks for the occurrence of prolonged flexion posture defined here as a deviation from the neutral position for more than a margin of $20^{\circ}$; the user is notified once this range is exceeded for longer than $1,5 \mathrm{sec}$. We decided on these values based on the settings adopted by Ribeiro et al. in their user test [27], and adjusted these iteratively to avoid producing too many notifications which could make these be ignored. It is further noted that for practical use such settings should be tailored to the user or could even be adaptively faded, to ensure reliance on feedback is gradually reduced. In this trial and error process we also found that double notifications could occur for the same episode of poor posture. To prevent this, the condition was added that no notifications have been sent to the user in the past 5 minutes to prevent fatigue/saturation. The above three parameters (deviation from neutral position, minimal duration of poor posture episode, and the minimum separation between two notifications) represent key choices that support the intended user experience. However, further testing in field conditions is needed to further optimize these choices and ensure a positive user experience and effectiveness in actual use.

When a sustained poor posture episode is detected the shirt notifies the user via both an audio signal and via vibration. In the notification bar of the Smartphone a visual icon and a notification is provided. We introduced 
this redundancy to ensure that the user notices the message even when not looking at the screen. On tapping this notification, the user is redirected to the BackUp application which prompts the user to record the activity they were engaged in when the sustained flexion was detected. It then presents to the user a choice of typical activities such as working at the desk, handling a patient, etc. as well as an option 'other'. Based on the user's responses over time the application compiles an overview of activities associated with poor posture episodes and presents relevant tips to the user per activity. The user interface supports two languages (English and Dutch).

\subsection{Behaviour change strategy}

Notifications by BackUp aim to increase user's awareness of their posture while they work. However, it may be more useful to help the user identify the causes of a poor posture episode and which actions can potentially lead to low back pain [20]. Further, as noted already, patients with low back pain have a diminished ability to actively control the movements of the low back due to disturbed intrinsic feedback mechanisms [5] Therefore it is also important to instruct them how to adjust their posture [20]. On detecting a poor posture episode, the smartphone will prompt the user for their activity thus drawing attention to its possible causes. The extra attention and effort it requires from the user is aimed at ensuring that knowledge about improving one's posture can become associated to a context/activity.

\section{Field Study 1: Initial Assessment of ACCEPTANCE, Motivation ANd Credibility}

We deployed BackUp in a hospital during the day shift, with nurses wearing and testing the prototype while carrying out their normal duties (see figure 7). The aim of this field study was a) to verify that the shirt can be worn comfortably and operated correctly in a realistic context b) to evaluate how willing nurses are to use BackUp and their experience of using it c) to evaluate the perceived potential of the system as a prevention and therapy aid.

\subsection{Participants}

We invited the nursing staff in a neighbouring clinic to participate in the evaluation using personal contacts and announcements at their workplace. Nurses participated voluntarily, without any monetary incentive. Respondents were screened with the following inclusion criteria: their job should not be sedentary, and they 
should not be suffering from low back pain.15 female nurses (ages 20-65) recruited in this way participated in the evaluation. Participants were informed about the study prior to their participation and provided informed consent before taking part.

\subsection{Study design}

We adopted a 1x2 within subjects design where each participant was exposed to two feedback strategies: a) vibration and audio notification as is common in most current posture monitoring systems and b) full feedback which next to the notifications of poor posture episodes requires from users to record the activity relating to the episode in order to provide them with a summative overview of activities that strain their low back. In both conditions, participants used the same shirt (medium size) and phone. Exposure to each feedback strategy lasted for an hour.

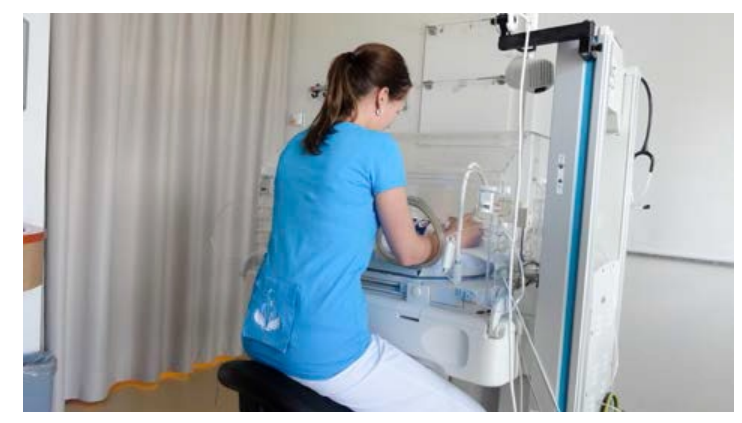

Figure 7. BackUp in use during the field test in the neonatal unit

\subsection{Procedure}

The researcher initialized and calibrated the application and adjusted the sensor patch position for the participant. Participants put the shirt on, carried a smartphone we provided to them with the BackUp application running and then engaged with their daily work. They wore the garment for two hours. The order of treatments (feedback strategies they experienced) was counter balanced. At the end of an hour in each of the two conditions, a questionnaire was handed out containing questions about the experience of the system.

\subsection{Measures}

Three validated questionnaires were used to evaluate different aspects of the participants' experience. Tapping on both user's reasoning and their feelings, the "credibility and expectancy" questionnaire [10] was used, to assess 
how much they believed BackUp can help improve low back conditions, measured by the credibility subscale, and to what extent they felt it can lead to improvements of low back conditions, measured by the expectancy subscale. This instrument emphasizes that what people rationally assess as the potential benefit of the method is different than what they feel this benefit might be [14]. The aggregate scores for credibility and expectancy can range from 3 to 27 . The CEQ mixes two types of rating scales; expectations are rated from $0-100 \%$ and beliefs are rated directly in scales from 1 to 9 . Following the instructions in [10] for scoring participant responses, the percentage scores are transformed linearly to a figure from 1 to 9 (by dividing by 12,5 and adding 1). The three items measuring credibility are s up with a minimum score of 3 and a maximum score of 27; the same holds for the three items measuring expectancy.

The "Intrinsic Motivation Inventory" [40] is a multidimensional measurement device intended to assess participants' subjective experience related to a target activity; in this case wearing and using BackUp. This inventory has six subscales, each consisting of two or more 7-point rating scales (levels 1-7) and measuring respectively: interest/enjoyment, perceived competence, effort, value/usefulness, pressure and tension felt, and perceived choice while performing a given activity, thus yielding six subscale scores. The interest/enjoyment subscale is considered the most essential self-report measure of intrinsic motivation; although the overall questionnaire is called the Intrinsic Motivation Inventory, it is only this one subscale that assesses intrinsic motivation [40]. The perceived competence concept is theorized to be a positive predictor of both self-reported and behavioural measures of intrinsic motivation, and pressure/tension is theorized to be a negative predictor of intrinsic motivation. The value/usefulness subscale is used in internalization studies [28] the idea being that people internalize and become self-regulating with respect to activities that they experience as useful or valuable for themselves.

The UTAUT questionnaire [34] was used with minor textual adaptations to apply to a wearable tracking device as opposed to a general information system which is what the phrasing of the original questionnaire implies. UTAUT is a model that attempts to predict people's intention to use a particular technology, from several factors that have been argued by researchers to predict this: e.g., ease of use, usefulness, and social norms. The questionnaire relating to UTAUT includes eight rating scales of seven levels (1-7). 
Finally, we asked three open questions regarding how comfortable the prototype is, how clear the feedback is and how easy it is to use.

\subsection{Results}

The system functioned without problems throughout the study. The participating nurses could all use it independently after a brief explanation of its operation and they were all able to complete the test successfully. The system reported a varying number of notifications for each participant $(M=12,4, S D=9,09)$.

The reported credibility of the system ranged from 19.1 to 24.9 ; credibility and did not differ significantly between the full feedback condition $(M=22, S D=2.89)$ compared to when a notification only was provided $(M=21,7, S D=2,64)$. Similarly, expectancy scores ranged from 16.2 to 22.6 , and were similar for the full feedback $(M=21.17, S D=2,64)$ and the notification only $(M=18.15, S D=2.63)$ conditions.

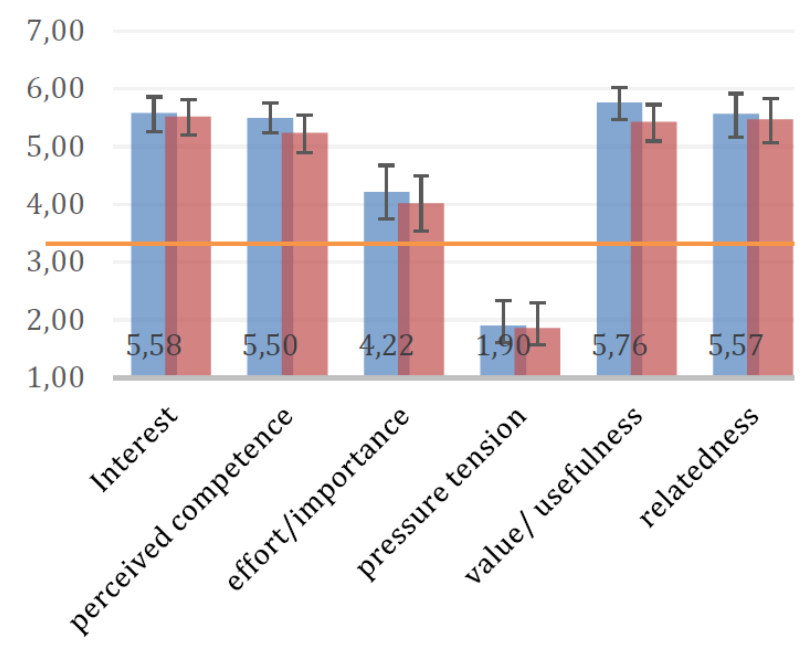

Figure 8. Intrinsic motivation graph for each construct for with and without the awareness building interaction; for case 1 and 2 ratings for interest, perceived competence, value/usefulness, and relatedness are all above the neutral score, pressure and tension are below it.

The scores for the Intrinsic Motivation Inventory [40] are shown in figure 8 plotted against the neutral score of 3,5. No significant differences were found between the two feedback conditions (t-tests were carried out to compare average ratings on all sub scales) which means that the addition of self-report and tips regarding the activity associated with the poor posture episode does not improve motivation. However, by the same token, is 
not shown to have adverse effects, something one might have expected given of the extra attention and effort that this feedback strategy imposes upon users.

We analysed interview transcripts thematically [4] using NVivo. Two coders sorted the data independently and formed nodes with similar meaning, which they then discussed to resolve any differences and ambiguities. Eventually we concluded with 16 positive notions about how comfortable the system was to wear compared to 2 negative notions.

The qualitative results suggest that that the shirt is comfortable (P1, P2, P6, P8, P10, P11) and easy to wear (P2, P3, P4, P5, P6, P8, P10). For example: P6 wrote "the wearability is good, no downsides". Participant P3 mentioned "I would like to use this during an intubation, because this is a posture that's hard for your low back." They reported as a positive element that they could not feel the shirt at all and that it did not restrict their movements; which seems to suggest what is the required level of wearability / comfort for smart garments: "You do not notice you wear it...Great!" (P3) or "The shirt is comfortable, you don't feel anything on your back" (P6) and "You don't notice you are wearing this shirt, only when there is a beep because of a bad posture" (P4). The last remark could also suggest that frequent feedback (whether audio or vibrotactile) may compromise the perceived comfort/wearability of the shirt by demanding too much attention (P2). Comfort/unobtrusiveness exhibits, at least in this case, a cross-modal nature.

The negative notions included that the shirt did not fit well P1, who normally wears 'small' size shirt rather than medium which we provided, and P7 found our prototype too long. However, this reflects the limitations of the study for which only one size was available rather than something inherent to the design concept. The second negative notion concerned comfort. P3 commented that the shirt was too warm as she wore it on top of her own shirt and P10 found wearing an 'extra' shirt warm. Considering that the shirt was made from the same material and had similar styling as the standard issue T-Shirts they wear under their tunic, this comment brings to the fore a fine difference between a deployment in a research study and actual use for the case of smart garments. Were the device her own she would perhaps not have hesitated to wear it directly on her skin. It also reminds us, that while developing the shirt we assume it will be worn in a certain way (in close contact to the body); but users already in a field study deviate from these assumptions in unforeseen ways. For a posture monitoring wearable to 
work well in real life, relevant instructions have to be provided to the nurses and the robustness of the sensing to different ways of wearing the shirt (under or on top of other garments) would need to be further established.

One participant noted that the wearability is very good, but the LED indicators on the modules raised questions from her social surroundings (P2). While we relied on these LED indicators for debugging during development and for technical inspection during this study, we had neglected to disable them in this study. More generally, it appears sensible to make conservative use of visual elements to support interaction with smart clothing, at least in the context of posture monitoring, as they increase the obtrusiveness of the device making it more remarkable than it needs to be. Another suggestion by P11 was in favour of a sleeveless version.

Participants commented that the feedback was very clear (P2, P3, P4, P5, P7, P10, P11), that the system is easy to use (P3, P8, P9), requires no explanation (P8) and is 'user friendly' (P3). Participants commented positively regarding the value of full feedback in changing posture: "tracking my activities has more effect for $m e$ " considering that relating posture to different activities helps them improve it. They argued that they could ignore a notification but appreciated the resulting awareness "for me the process of becoming aware, in which activities I had a bad posture made it more attractive". Self-awareness is known to be one of the major motivators for the use of self-tracking technologies [16] and these comments suggest that the full feedback strategy could support this purpose better than simple notifications. This function in itself was found valuable; as P3 commented: "it helps you correct your posture even though at on the moment itself you don't feel any pain or obstruction; it is good to know that you do not have an optimal posture".

Regarding the two feedback strategies, participant P5 found the extended feedback unnecessary for her, though she also mentioned that it helped her become more aware of her posture. P6 would have liked a finer grain classification of straining postures to relate to her activities, showing an interest to invest even more effort in correcting her posture; she specifically expressed her wish to know more about her posture by wearing the device longer and to understand how different her posture is at 8:00 am when her shift starts versus later in the afternoon P2 commented how a better posture was not possible in some occasions; while such contextual judgments are difficult to make given a wearable device, it is hoped that flagging such occurrences can help trigger reflection 
and perhaps redesign of the work situations. However, in long-term use one might consider supporting customization by users to disable the notifications in specific situations.

\section{FiELd STUdY 2: GAUGING THE EFFECTIVENESS OF BACKUP}

A second field study was carried out to establish that using the system influences posture behaviour positively. Furthermore, we wished to compare the impact of the two different feedback strategies on posture. Since posture behaviour is expected to be sensitive to social and environmental influences, this study was conducted in the field while participants were engaged in their daily work at their actual working environment.

\subsection{Participants}

The recruitment and screening process were identical to the first study. 13 female nurses aged from 22 to 61 years $(M=39.77, S D=13.63)$ from a different hospital than the first study participated. Nine of them were working in neonatal intensive care unit. The remaining four nurses were working for home care. Participants provided informed consent on the first meeting with the researcher.

\subsection{Research design}

For the field study, a triangulated mixed methods [8] approach was followed, which combines quantitative and qualitative data collection. A within-subject design was applied where participants followed a continuous four-phased (A-B-A-C) treatment implementation and replication in real working context. A-B-A-C design is a common adaption of the A-B-A-B reversal design [19] used to compare different treatments (feedback strategies in our case). In reversal design, the researcher removes the treatment and reverses back to baseline after the first treatment phase, to demonstrate a functional relationship between treatment and behaviour. In order to evaluate more than one form of feedback we replaced the last treatment phase into a second treatment $\mathrm{C}$.

- Condition A: Participants went on with their daily work and no notification and no interaction with the system was possible. The system tracked their posture to obtain a baseline measurement.

- Condition B: In this treatment condition, participants only received notification of poor posture episodes that included sound and vibration without any other interaction. 
- Condition C: Participants received the full feedback which combined notifications of poor posture episodes with self-recording of related activities and a daily overview of activities that could cause low back pain (Figure 8) in the application.

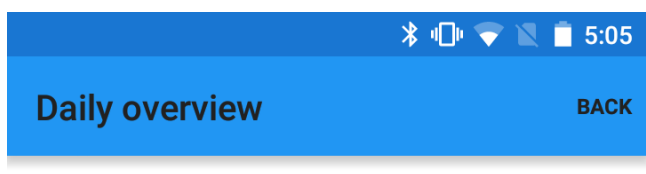

User Overview of activities that can cause lower back pain

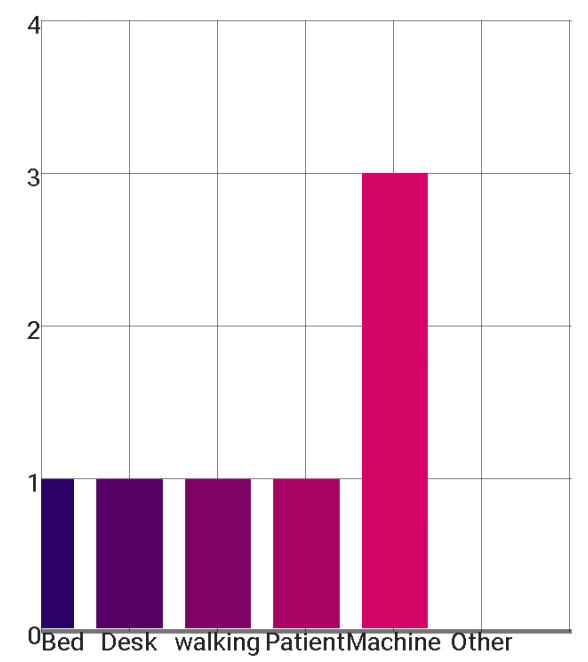

Figure 9. Daily overview of activities that can cause low back pain

\subsection{Procedures}

The participants used the same shirt (medium size) and smartphone in all conditions. The researcher adjusted the sensor placement and helped initialize the application and calibrated it for each participant and remained available in a break room of the hospital in case unexpected problems would arise, but remained out of sight and did not interact with participants other than to explain the study procedures at the start of each phase. The fourphased evaluation (A-B-A-C) was conducted in the course of one (different) day for each participant. The first baseline phase (A) lasted for 30 minutes and the other three phases took 60 minutes respectively. Questionnaires were administered at two moments after phases B and C. At the end of the session and after filling the second round of questionnaires participants were interviewed individually. 


\subsection{Measures}

The following measures were taken

- Treatment expectancy and credibility. After phases B and C, nurses completed the CEQ questionnaire as in the first study. The repeated measures were aimed at comparing the two feedback strategies.

- The Intrinsic motivation inventory was used after B and C to compare the two feedback strategies.

- Acceptance and use of technology. At the end of the session (after C), nurses filled in an adapted version of the UTAUT questionnaire [34] in order to measure acceptance of the system involving all its feedback possibilities.

- Occurrence of poor posture episodes. The effectiveness of BackUp was assessed by analysing posture data. An occurrence of a poor posture episode was considered to be a deviation of the curvature of the spine from the calibration values for a specific participant for more than 20 degrees and for longer than 1.5 consecutive seconds, as these were the threshold values used to notify participants of poor posture episodes.

- Semi-structured interview. Following the initial use of the system, the researcher interviewed nurses describing their experience and expressing their thoughts on the further development of this system. The interview was audio recorded and selectively transcribed.

\subsection{Results}

The quantitative data from the questionnaires was analysed with non-parametric test including Wilcoxon signed-rank test and the logged number of poor posture episodes using a one-way repeated measures ANOVA. As in the first study, qualitative data was analysed using thematic analysis focusing on three broad topical categories: reflections on the system, expected feedback, and motivation.

\subsection{Treatment expectancy and credibility}

Participants ratings in the treatment condition B for credibility $(M=20,85, S D=3,84)$ and expectancy $(M=17,48, S D=3,48)$ were similar to the ratings for credibility $(M=19,54, S D=4,72)$ and expectancy $(M=19,54$, $S D=4,5$ ) in the full feedback condition C. A Wilkoxon signed ranks test revealed no significant difference in 
nurses' outcome expectations and perceptions of credibility of treatment between the two treatment conditions at the 0.05 significance level: credibility $(W=27, N=11, p=0,23$, two-tailed); expectancy $(W=5, N=13, p=$ 0.87, two-tailed).

\subsection{Intrinsic Motivation}

Participants responded with scores above 4, for all subscales of the intrinsic motivation inventory, apart from the pressure/tension, which is a negative predictor and that received a score less than 4 (see table 2). A score of 4 in these scales would indicate a neutral attitude because questions were on 7-point scale ranging from 1 to 7. Notably, value/usefulness ranks the first among subscales, which indicates that participants were motivated to use the system and were able to perceive or experience the system as useful or valuable for themselves in improving their posture and avoiding low back pain. The interviews confirmed these attitudes regarding the perceived health value for motivation in long term like "If it can reduce my low back pain, I will use it in long term" (P1) and "The improvement in the muscle of low back and the increasing knowledge of the correct posture may motivate me to change in long term." (P10). Comparing the motivation scores did not reveal any differences for the two feedback conditions in any of the subscales (see table 2)

\begin{tabular}{|lcccc|l|}
\hline & \multicolumn{7}{c}{ Treatment B } & \multicolumn{2}{r|}{ Treatment C } & \\
\hline \multicolumn{1}{|c}{ Measure } & M & SD & M & SD & \multicolumn{1}{|c|}{ Signed ranks test (2-tailed) } \\
Interest/ Enjoyment & 5.28 & 0.95 & 5.23 & 1.13 & $\mathrm{~W}=36, \mathrm{~N}=12, \mathrm{p}=0.858$ \\
Effort/ Importance & 4.36 & 0.82 & 4.33 & 0.76 & $\mathrm{~W}=27.5 \mathrm{~N}=10, \mathrm{p}=1$ \\
Pressure/ Tension & 2.10 & 1.19 & 2.38 & 1.16 & $\mathrm{~W}=7 \mathrm{~N}=7, \mathrm{p}=0.522$ \\
Perceived Competence & 4.85 & 0.83 & 4.85 & 1.20 & $\mathrm{~W}=31 \mathrm{~N}=12, \mathrm{p}=0.878$ \\
Value/ Usefulness & 5.79 & 0.97 & 5.58 & 1.22 & $\mathrm{~W}=8.5 \mathrm{~N}=7, \mathrm{p}=0.778$ \\
Relatedness & 5.28 & 0.87 & 4.90 & 1.37 & $\mathrm{~W}=9.5, \mathrm{~N}=12, \mathrm{p}=0.412$ \\
\hline
\end{tabular}

Table 2. Subscale scores of intrinsic motivation inventory and comparison between the two feedback conditions 


\subsection{Technology Acceptance}

Participants seemed moderately positive towards the system overall as their UTAUT scores (see Figure 10). However, the large confidence intervals reflect how some participants even rated the system negatively. Effort expectancy and hedonic motivation were rated higher than neutral. Hedonic motivation can be linked to intrinsic motivation. However, the comments provided by all participants have focused exclusively on the functional purpose of the device rather than the aesthetic aspects of its design. The score of Effort expectancy illustrates that the system was easy to use for participants. On the other hand, the system was rated lowest in the social norms scale with some participants reporting a negative influence from their environment. According to P4 who was the only one to comment on social norms, "the work place does not always make it possible to correct your position". During the study the shirt attracted the attention of colleagues or patients due to the audio notifications; this attention may have contributed to feelings of negative social influence for some participants.

Through the interview, participants also indicated that the ease of use was an important requirement: "The application should be easy to use, especially for older colleagues." (P2).

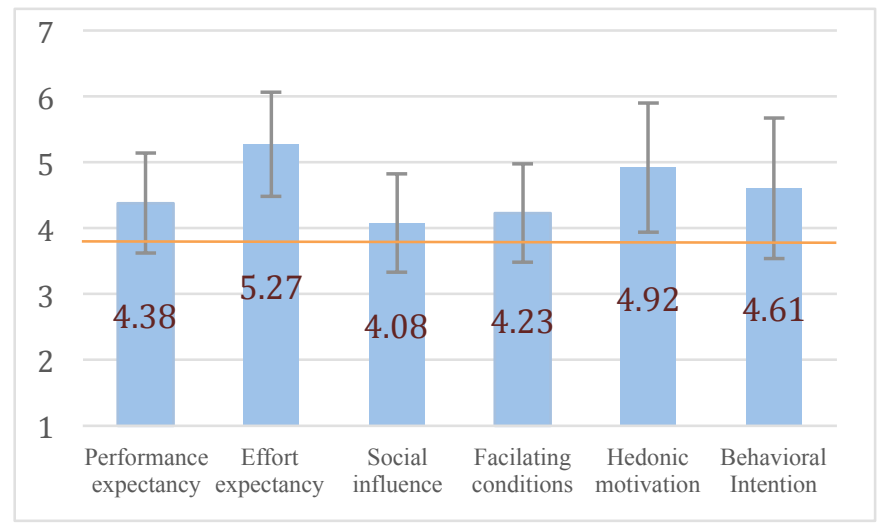

Figure 10. Average scores and $95 \%$ confidence intervals of technology acceptance for each construct

\section{$5.9 \quad$ Effectiveness of posture tracking}

A one-way repeated measures ANOVA was conducted comparing the effect of the feedback strategy on the number of occurrences of poor posture episodes across the four phases of the study. A significant effect of feedback strategy was found $F(3,12)=7,97, p<0,01$. Post-hoc analysis indicated that the number of poor posture occurrences was significantly higher in the initial baseline condition $(M=25.54, S D=12.49)$ and the withdrawal 
condition $(M=24.85, S D=12.78)$ compared to treatment B when receiving notifications $(M=22.08, S D=10.84)$ and to the treatment $\mathrm{C}$ when receiving the full feedback $(M=19.08, S D=12.18)$. The differences between occurrences of poor posture episodes in the two treatment conditions B and C were not significant. The overall trend has a negative slope, which may indicate a learning effect: participants gradually improve their posture. Future research could examine whether such learning takes place with longer use of the system and confirmed through follow up measurements after withdrawing it. Occurrences of posture notifications ranged widely in each condition due to the different workload of per participant, but in general they show similar trend as described above.

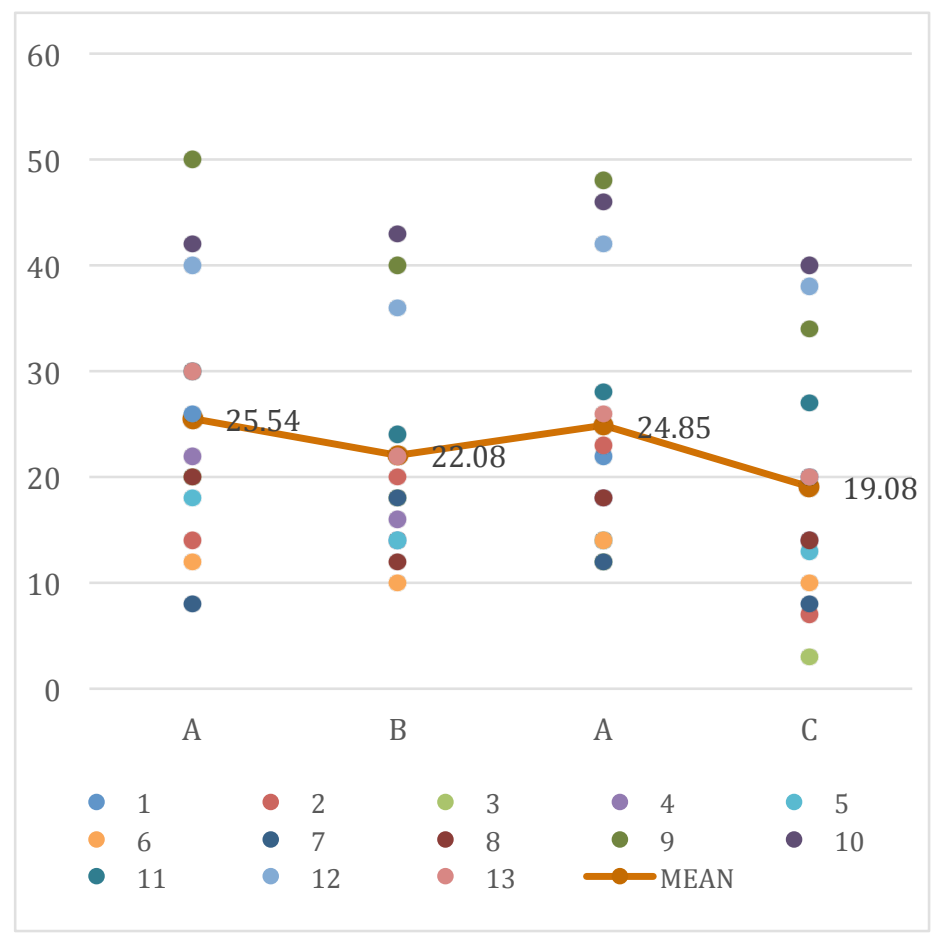

Figure 11. Occurrences of poor posture episodes per participant in the four phases of the study

\subsection{Qualitative data analysis}

Participants were not completely satisfied with the posture notifications and responding to requests for focal interactions to associate activities to sustained poor posture occurrences was not always convenient for them due to their work. They expressed the following requirements regarding posture feedback:

- Participants indicated the need for some normative feedback that would tell them how good or bad their posture behaviour is. Especially one participant ( $\mathrm{P} 4)$ would rather receive some positive reinforcement rather than just negative notifications. 
- Participants would like to receive a summary of their posture behaviour close in time to the occurrence of sustained poor posture events. Parameters they would like to know about include angle, latency, and direction, etc. "I want to know what I always do wrong too much bending left or right" (P9) "I want to know what is bad for my low back" (P5).

- Participants would like to be given prescriptive feedback as to what is a better way to do a specific task: "Giving tips for better posture according to my situation" (P3), and concrete advice for how to improve their posture and how to adjust workplace (P5).

- Participants reported positive attitudes towards tracking their progress in the long term (P3, P9, P10, P11) and suggested that confirmation of improvements could motivate them to continue using the system further (P4).

Common in the above is the desire to receive feedback that is based on individual circumstances. On the other hand, opinions were mixed regarding sharing posture data with others. E.g., P11 was positive wanting to "see if it (my progress) matches others and I can learn from others", or P9 wanting to "see what others can do in terms of posture". On the other hand, P10 was sceptical about the possibility of sharing posture data :, "I do not want to share the data with others because everyone is different".

\section{DISCUSSION}

BackUp operated successfully in field conditions for field studies lasting a few consecutive hours. Participants ratings along the various measures taken were generally encouraging regarding its credibility to support posture correction and about their motivation to use it for this purpose. Contrary to our expectations, the addition of focal interaction to increase awareness of activities relating to posture and providing tips for improvement, did not significantly influence the participants' attitude and thoughts regarding the BackUp system during the limited exposure they had to the system. On the upside the extra effort / interruption does not seem to annoy users; on the downside the expected self-awareness regarding posture was not manifested in the logged posture data. To ensure that the system remains unobtrusive when used for long periods customizable or context 
sensitive delivery of notifications to fit the working environment and workload could address the user in a more subtle and flexible way, and thus ensure the system can be experienced as unobtrusive over the long term.

The second study showed that both treatments had a positive impact on posture at least in the short term. Unfortunately, from the current results, it cannot be determined which feedback strategy is the most effective for changing behaviour (simple notifications versus full feedback). Future research could pursue a comparison of different feedback strategies, and also examine their relative effectiveness over longer periods of testing. This appears necessary, as it is well known that different feedback mechanisms can have a profound effect on the effectiveness of the rehabilitation [26]

As a limitation of our results it is noted that the participant sample is clearly not representative of male nurses, which limits the generalizability of the results; however, given that the majority of nurses in the Netherlands are female [38], it ensures at least the relevance of our results for addressing low back pain within this population.

The outcomes demonstrated are short-term and it is noteworthy that in the withdrawal condition (after having received notifications for an hour) the number of poor posture episodes was similar to the initial baseline (see figure 11). As one might expect, users appeared to rely on the extrinsic feedback for avoiding a prolonged flexion posture and that learning to do so unaided by technology can take longer.

This test examined the potential of BackUp when this is used as a prevention tool - participants were all healthy without any low back problems. Future research should examine whether low back patients also appreciate it in a similar way and whether it helps them during rehabilitation and to prevent relapse. Given that this kind of tracking technology can be worn every day, it is interesting to examine user experiences over sustained usage lasting several weeks. This would counter novelty effects and more importantly, it would test whether users start to ignore the system or get annoyed by repetition. While we expect this to be so, improvements in the scheduling of reminders and prompts could help address these issues. Based on Weering's work [9] we can expect improvements from personalising notifications based on the time of the day and the intensity of the movements at that time or over time. The response of the user may be better when (s)he can choose the time of the day that the messages are delivered [18]. Also allowing for customized settings for delivery 
of notifications (personal bandwidth settings for the inclination at which the posture is recognized as being adverse, personal settings for how long an adverse posture needs to be maintained before it will notify the user) is a way to address the user in a more personal way (the duration and deviation of neutral in which a posture can be identified as adverse is different for different users, different pathologies, different working contexts, etc.). For example, the duration of 1,5 seconds used here as a notification criterion may be too short for persons who do not suffer from back pain yet (prevention).

Regarding the industrial design of the shirt, several improvements can be made to ensure practical use and manufacturing. For example, the T-Shirt is made to be washable, but the battery still needs to be removed and charged separately. For a seamless user experience the whole product should be washable and conveniently chargeable, for example through inductive charging. Furthermore, BackUp has many components connected together by an embroidered circuit. For industrial production and professional use, it would be necessary to custom-print circuit boards so as to minimize production costs and the footprint of the electronics.

So far both behaviour change strategies implemented are based on informing users and our tests established how this affects their attitudes towards the system and postural behaviour change positively. However, much of postural behaviour is habitual and does not necessarily follow conscious and rational reasoning; dual process theories also note the importance of heuristics and social influence [6]. Related approaches to behaviour change would be worth exploring in order to achieve a sustainable behaviour change. We note that during the field studies, other nurses, who were not participating in the study, expressed their wish to try the system as they saw their colleagues using it. Since nurses in a hospital, at least in the one we examined, form an existing social network with their colleagues, adding a social influence layer to the behaviour change strategy may be acceptable and meaningful for them. Nevertheless, qualitative data collected during this study was ambivalent with regards to sharing posture data with colleagues.

\section{GENERAL CHALLENGES FOR DEVELOPING SMART GARMENTS FOR PREVENTION AND} REHABILITATION

BackUp is the first smart garment created to monitor low back posture; it is an experimental prototype that so far looks promising for its potential to change behaviour and to be accepted by nurses, though further 
development and longer-term testing are still required before it can be used widely. BackUp was designed for nurses for whom occupational low back pain is a serious issue justifying the need for the intervention and for whom the use of uniforms makes it easy to implement interventions based on smart garments in practice. For example, the hospital could purchase posture-monitoring T-shirts and supply them to nurses. Similar but different challenges may be associated with occupational low back prevention for other professions: e.g., for construction workers one might be less inclined to ask them to wear a T-shirt under their uniform, but rather would place the sensing technology on the overalls they use. We note how a generic product/garment to support posture tracking at work may be elusive: requirements for the sensor placement, the accuracy and reliability of posture tracking appear to be specific to the user group, their activities and the clothes they wear. Information workers who need to maintain a good posture may also have different requirements, different ranges of movement to monitor, and different requirements regarding appearance. For workers sitting in front of a workstation, it is clear that there are also more possibilities for providing feedback by instrumenting the environment and using desktop devices in the vicinity of the user rather than directly on the body or through audio notifications provided by a mobile device.

As designers we have not strived for a general solution for preventing low back problems of the general consumer. Rather, we have focused on a specific target group with a pronounced need for posture monitoring and suggested a solution fitting this context and worked together with target users to develop the solution. This more contextualized approach favours a design driven investigation as opposed to, e.g. establishing general persuasion and measurement principles before applying them in the specific context. Below we note some of the lessons learnt regarding the industrial design of posture tracking that could help in designing solutions in this domain.

Fitting sensing technology on clothes rather than using contraptions like belts or clip-on devices, offers several advantages such as a closer fit to the body, comfort, and unobtrusiveness. Tailoring and fitting the garment helps ensure accurate positioning of the sensors, where the user can put the device on without the aid of a professional. In this sense, tracking garments may have an important role to play in everyday life beyond the case of low back pain prevention, but considering biofeedback applications in general. On the other hand, designing smart clothing presents several design challenges. During the development of BackUp these have been addressed iteratively with close cooperation with experts in movement sciences, designers of work clothes, and nurses. This 
design process presented has revolved around a few key challenges that apply generally to the design of smart garments for biofeedback applications:

Biosignal sensing using wearable technology requires accurate positioning of sensors. Clothes typically shift over the skin, so a main challenge for designers of any type of smart garments (e.g. shirts, sleeves, socks, etc.) is to ensure that the sensors will be positioned at the right place and their readings are correctly interpreted. The approach proposed with BackUp is to manufacture a few default sizes (large, medium, small, etc.) of a general garment, which the user can tailor-fit and calibrate for more precise and individual positioning of sensors. This approach has clear limits as it provides only a coarse classification of human bodies. An emerging possibility concerns the development of ultra-personalized systems and services, where the garment is tailored to the body of a specific individual [2]. Related technologies and production processes are only starting to emerge, but may soon represent a major enabler for the wider application of smart garments in therapy and healthy living applications.

A recurring challenge that applies to all smart garments used for such purposes is how to connect hard electronics to the garment, potentially featuring some soft (textile) electronics. Some parts (e.g., the battery) may need to be detachable, or sometimes the whole microcontroller. The connection between the soft and hard electronics is a vulnerable part of the design, where wear and tear can reduce robustness and durability. Developing textile sensors to replace hard components may mitigate this problem, eventually only having a single detachable component (e.g., the microprocessor). In the case of posture correction, rather than relying on inertial measurement units or accelerometers, one could attempt to measure the curvature of the lumbar spine directly through the deformations of textile components. Advances in materials and e-textiles could make such innovations possible, e.g., see Fleury et al [11].

Garments lend themselves particularly for prevention and training that spans over days, weeks, or even longer; though in the case of BackUp such a sustained use is still the subject of future research. In this context next to the industrial design of smart garments, designing a suitable behaviour change strategy is one of the key challenges for this research field. Current self-tracking solutions seem to be largely restricted to simple alerts (especially in the case of posture monitoring), which may not always be the optimal solution. Feedback strategies that include tailoring the bandwidth of movements that are considered allowable, varying the frequency of alerts 
provided, allowing social influence strategies, are some strategies that are known to improve the effectiveness of feedback during rehabilitation [26]. It may be expected that these should apply for motor learning also for prevention and healthy living applications, though this still needs to be demonstrated empirically. The short-term evaluation presented here, has not provided the expected evidence regarding the self-efficacy increasing interactions; rather than abandoning and modifying this strategy evaluating it in more prolonged and larger scale studies may be useful as it may well pay off in the long term.

Turning our attention to the design process followed to address these challenges, there are a few key elements that are useful to emphasize regarding how to design smart garments for prevention and rehabilitation. For example, the design process involved substantial experimentation and trial and error, (including several smallscale explorations nested within the iterations that were described above). This is because disparate requirements regarding comfort, appearance, accuracy, and technology integration are closely coupled to each other and many of these are not understood a priori to allow a more linear specify-prototype-test approach. While intense and exploratory prototyping is generally accepted as beneficial in most areas of interaction design, designing for garments makes this need for a holistic treatment of concerns in tight iterations even more pronounced. Especially, emphasizing on aesthetic qualities is not only a concern that can be left for the final stages of the design process but is required for ensuring participation of users in tests (as they had to wear the garment at work) and their ability to provide relevant feedback on wearability. Furthermore, the need to prototype and test has brought up the need for anthropometric data that was not readily available, and which could not be obtained correctly without the participation of health scientists. While the emphasis on integration and appearance emphasizes on the craft skills of the designer, ensuring the effectiveness of the solution has also emphasized an empiricist approach to research through design, following a mixed methods approach for collecting and analysing data, and the active involvement of a multi-disciplinary team.

\section{CONCLUSION}

The contribution of this article is to demonstrate the feasibility of low back posture monitoring for the prevention and treatment low back pain using smart garments. It introduces BackUp a purpose-specific solution designed for nurses: BackUp is a T-shirt with a tight fit and patches that allow personalized sensor placement for 
monitoring the low back posture. Furthermore, the paper documents an iterative design process, which addressed a number of complex requirements regarding accuracy, appearance, comfort and design for behaviour change.

The device improves upon the current state of the art in that it tracks low back posture (the curvature of the lumbar spine) rather than a coarse measure of posture/inclination (how upright the user is) as current commercial devices and state of the art technologies do. The paper contributes to the literature in wearable biofeedback systems by highlighting general challenges involved in these domains and presenting the solutions invented to measure spinal posture from sensors attached to a garment as a resource for technology designers engaging in this domain.

A behaviour change strategy built on increasing self-awareness of low back posture and educating users how to correct it has been presented. BackUp has been evaluated in a field study, adding to existing literature on low back posture monitoring where there is a scarcity of such studies. Further, the paper has exemplified how an adopted A-B-A-C reversal design using mixed methods to collect both quantitative and qualitative data can be used to evaluate self-tracking solutions. This approach common in behaviour modification studies is relatively underutilized in the broader field of persuasive technologies and could be useful when studying self-tracking technologies. The evaluation of BackUp has shown that using the system helps decrease of occurrences of undesired posture behaviour in the short term. Further research is needed to improve the robustness of the system and to develop suitable behaviour change strategies in order to support posture correction over longer periods of time.

\section{ACKNOWLEDGEMENTS}

Wang Qi gratefully acknowledges funding from the Chinese Scholarship Council. The nurses of Sint Katharina Hospital Eindhoven and Maxima Medisch Centrum Eindhoven are thanked for their participation. Marina Toeters is thanked for her support and expertise in designing clothing for nurses. Han Hamers is thanked for the assistance in designing the embroidered electronics to enable mass production. The students of Hasselt University (BE) are thanked for their assistance in the measurements of intervertebral distances. 


\section{REFERENCES}

1. Marc van Almkerk, Bart L. Bierling, Nono Leermakers, Jeroen Vinken, and Annick A. A. Timmermans. 2015. Improving posture and sitting behaviour through tactile and visual feedback in a sedentary environment. 4570-4573. https://doi.org/10.1109/EMBC.2015.7319411

2. Martijn ten Bhomer, Oscar Tomico, and Stephan Wensveen. 2016. Designing ultra-personalised embodied smart textile services for well-being. Retrieved August 28, 2016 from https://www.mtbhomer.com/downloads/publications/tenbhomer-2015.pdf

3. Katia M. Black, Angela Lis, and Margareta Nordin. 2011. Association between sitting and occupational low back pain (lbp). Revista Ação Ergonômica 1, 3. Retrieved September 23, 2015 from http://www.abergo.org.br/revista/index.php/ae/article/view/26

4. Virginia Braun and Victoria Clarke. 2006. Using thematic analysis in psychology. Qualitative Research in Psychology 3, 2: 77-101. https://doi.org/10.1191/1478088706qp063oa

5. Simon Brumagne, Paul Cordo, and Sabine Verschueren. 2004. Proprioceptive weighting changes in persons with low back pain and elderly persons during upright standing. Neuroscience Letters 366, 1: 6366. https://doi.org/10.1016/j.neulet.2004.05.013

6. Robert B. Cialdini and Melanie R. Trost. 1998. Social influence: Social norms, conformity and compliance. Retrieved August 28, 2016 from http://psycnet.apa.org/psycinfo/1998-07091-021

7. Tobias Consmüller, Antonius Rohlmann, Daniel Weinland, Claudia Druschel, Georg N. Duda, and William R. Taylor. 2012. Comparative evaluation of a novel measurement tool to assess lumbar spine posture and range of motion. European Spine Journal 21, 11: 2170-2180.

8. John W. Creswell and Vicki L. Plano Clark. 2007. Designing and conducting mixed methods research. Retrieved August 24, 2016 from http://onlinelibrary.wiley.com/doi/10.1111/j.17536405.2007.00097.x/full

9. Marit GH Dekker-van Weering, Miriam MR Vollenbroek-Hutten, and Hermie J. Hermens. 2012. Do personalized feedback messages about activity patterns stimulate patients with chronic low back pain to 
change their activity behaviour on a short-term notice? Applied psychophysiology and biofeedback 37, 2: 8189.

10. Grant J. Devilly and Thomas D. Borkovec. 2000. Psychometric properties of the credibility/expectancy questionnaire. Journal of behavior therapy and experimental psychiatry 31, 2: 73-86.

11. Amanda Fleury, Maddy Sugar, and Tom Chau. 2015. E-textiles in clinical rehabilitation: a scoping review. Electronics 4, 1: 173-203.

12. Sonja Freitag, Isabell Fincke-Junod, Rachida Seddouki, Madeleine Dulon, Ingo Hermanns, Jan Felix Kersten, Tore J. Larsson, and Albert Nienhaus. 2012. Frequent bending — an underestimated burden in nursing professions. Annals of occupational hygiene 56, 6: 697-707.

13. Gary Hsieh, Kenneth Wood, and Abigail Sellen. 2006. Peripheral display of digital handwritten notes. In Proceedings of the SIGCHI conference on Human Factors in computing systems, 285-288. Retrieved August 28, 2016 from http://dl.acm.org/citation.cfm?id=1124815

14. Alan E. Kazdin. 1979. Therapy outcome questions requiring control of credibility and treatmentgenerated expectancies. Behavior Therapy 10, 1: 81-93.

15. Peter Kent, Robert Laird, and Terry Haines. 2015. The effect of changing movement and posture using motion-sensor biofeedback, versus guidelines-based care, on the clinical outcomes of people with subacute or chronic low back pain-a multicentre, cluster-randomised, placebo-controlled, pilot trial. $B M C$ Musculoskeletal Disorders 16: 131. https://doi.org/10.1186/s12891-015-0591-5

16. Ian Li, Anind K. Dey, and Jodi Forlizzi. 2011. Understanding My Data, Myself: Supporting Selfreflection with Ubicomp Technologies. In Proceedings of the 13th International Conference on Ubiquitous Computing (UbiComp '11), 405-414. https://doi.org/10.1145/2030112.2030166

17. Angela Maria Lis, Katia M. Black, Hayley Korn, and Margareta Nordin. 2007. Association between sitting and occupational LBP. European Spine Journal 16, 2: 283-298.

18. Panos Markopoulos, Nikolaos Batalas, and Annick Timmermans. 2015. On the use of personalization to enhance compliance in experience sampling. In Proceedings of the European Conference 
on Cognitive Ergonomics 2015, 15. Retrieved August 25, 2016 from http://dl.acm.org/citation.cfm?id=2788427

19. Raymond Miltenberger. 2011. Behavior modification: Principles and procedures. Cengage

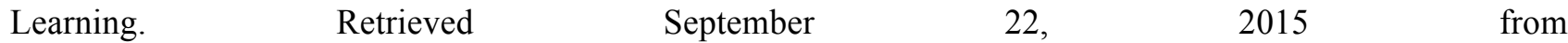
https://books.google.nl/books?hl=en\&lr=\&id=je0IAAAAQBAJ\&oi=fnd\&pg=PR4\&dq=Behavior+modificati on + Principles + and + Procedures\&ots $=$ NzukMKSivs\&sig $=$ hrPmjdERiVoK-i6V2x4oYQN1AEM

20. R. Miltenberger and others. 1999. Understanding problem behaviors through functional assessment. Challenging behavior of persons with mental health disorders and severe developmental disabilities: $215-235$.

21. Joseph W. Newbold, Nadia Bianchi-Berthouze, Nicolas E. Gold, Ana Tajadura-Jiménez, and Amanda CdC Williams. 2016. Musically informed sonification for chronic pain rehabilitation: Facilitating progress \& avoiding over-doing. In Proceedings of the 2016 CHI Conference on Human Factors in Computing Systems, 5698-5703. Retrieved from http://dl.acm.org/citation.cfm?id=2858302

22. Kieran O’Sullivan, Leonard O'Sullivan, Peter O'Sullivan, and Wim Dankaerts. 2013. Investigating the effect of real-time spinal postural biofeedback on seated discomfort in people with nonspecific chronic low back pain. Ergonomics 56, 8: 1315-1325.

23. Robert J. Parkinson, Tyson AC Beach, and Jack P. Callaghan. 2004. The time-varying response of the in vivo lumbar spine to dynamic repetitive flexion. Clinical Biomechanics 19, 4: 330-336.

24. Stéphane Poitras, Régis Blais, Bonnie Swaine, and Michel Rossignol. 2005. Management of Work-Related Low Back Pain: A Population-Based Survey of Physical Therapists. Physical Therapy 85, 11: $1168-1181$

25. Laura Punnett, Lawrence J. Fine, W. Monroe Keyserling, Gary D. Herrin, and Don B. Chaffin. 1991. Back disorders and nonneutral trunk postures of automobile assembly workers. Scandinavian journal of work, environment \& health: 337-346. 
26. Daniel Cury Ribeiro, Gisela Sole, J. Haxby Abbott, and Stephan Milosavljevic. 2011. Extrinsic feedback and management of low back pain: A critical review of the literature. Manual Therapy 16, 3: 231239. https://doi.org/10.1016/j.math.2010.12.001

27. Daniel Cury Ribeiro, Gisela Sole, J. Haxby Abbott, and Stephan Milosavljevic. 2014. The effectiveness of a lumbopelvic monitor and feedback device to change postural behavior: a feasibility randomized controlled trial. The Journal of Orthopaedic and Sports Physical Therapy 44, 9: 702-711. https://doi.org/10.2519/jospt.2014.5009

28. Richard M. Ryan and Edward L. Deci. 2000. Self-determination theory and the facilitation of intrinsic motivation, social development, and well-being. American psychologist 55, 1: 68.

29. Jaemyung Shin, Bumsoo Kang, Taiwoo Park, Jina Huh, Jinhan Kim, and Junehwa Song. 2016. BeUpright: Posture Correction Using Relational Norm Intervention. In Proceedings of the 2016 CHI Conference on Human Factors in Computing Systems, 6040-6052. Retrieved August 25, 2016 from http://dl.acm.org/citation.cfm?id=2858561

30. A. Timmermans, R. Bootsman, T. Matheve, and P. Markopoulos. Anthropometric Parameters for Sensor Placement in Wearable Technologies at the Trunk. Retrieved September 22, 2015 from http://biomedicasummit.com/Biomedica2015/pdf/poster/M06.pdf

31. Linda R. Van Dillen, Shirley A. Sahrmann, and Joanne M. Wagner. 2005. Classification, intervention, and outcomes for a person with lumbar rotation with flexion syndrome. Physical Therapy 85, 4: $336-351$.

32. Wannes Van Hoof, Koen Volkaerts, Kieran O'Sullivan, Sabine Verschueren, and Wim Dankaerts. 2012. Comparing lower lumbar kinematics in cyclists with low back pain (flexion pattern) versus asymptomatic controls - field study using a wireless posture monitoring system. Manual Therapy 17, 4: 312317. https://doi.org/10.1016/j.math.2012.02.012

33. Peter H. Veltink, Hans BJ Bussman, Frank Koelma, Henry M. Franken, Wim LJ Martens, and Rob C. Lummel. 1993. The feasibility of posture and movement detection by accelerometry. Retrieved September 22, 2015 from http://doc.utwente.n1/16744 
34. Viswanath Venkatesh, Michael G. Morris, Gordon B. Davis, and Fred D. Davis. 2003. User Acceptance of Information Technology: Toward a Unified View. MIS Quarterly 27, 3: 425-478.

35. Qi Wang, Panos Markopoulos, and Wei Chen. 2014. Smart rehabilitation garment design for armhand training. In Proceedings of the 8th International Conference on Pervasive Computing Technologies for Healthcare, 328-330. Retrieved September 22, 2015 from http://dl.acm.org/citation.cfm?id=2686965

36. Qi Wang, Panos Markopoulos, Bin Yu, Wei Chen, and Annick Timmermans. 2017. Interactive wearable systems for upper body rehabilitation: a systematic review. Journal of neuroengineering and rehabilitation 14, 1: 20.

37. Christina M. Wenig, Carsten O. Schmidt, Thomas Kohlmann, and Bernd Schweikert. 2009. Costs of back pain in Germany. European Journal of Pain 13, 3: 280-286.

38. Margaret Whittock and Laurence Leonard. 2003. Stepping outside the stereotype. A pilot study of the motivations and experiences of males in the nursing profession. Journal of nursing management 11, 4: $242-249$.

39. Ying Jean Zheng and John B. Morrell. 2010. Cognitive load assessment of a vibrotactile posture feedback chair. In Proceedings of the Human Factors and Ergonomics Society Annual Meeting, 1214-1218. Retrieved August 24, 2016 from http://pro.sagepub.com/content/54/15/1214.short

40. Intrinsic Motivation Inventory (IMI). selfdeterminationtheory.org. Retrieved September 22, 2015 from http://www.selfdeterminationtheory.org/intrinsic-motivation-inventory/ 